\title{
Role of RHOC in evaluating an adverse prognosis in patients with glioma and its potential prognostic value
}

\author{
BO ZHANG $^{1 *}$, JIAWEI YAO $^{2 *}$, XIAOYU LIAN ${ }^{3 *}$, BINFENG LIU $^{3}$, YANBIAO WANG $^{3}$, HONGBO WANG $^{1}$,

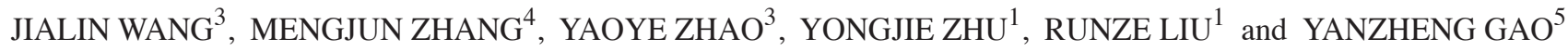 \\ ${ }^{1}$ Henan University People's Hospital, Henan Provincial People's Hospital, Zhengzhou, Henan 450003; \\ ${ }^{2}$ Department of Neurosurgery, First Affiliated Hospital of Harbin Medical University, Harbin, Heilongjiang 150001; \\ ${ }^{3}$ Department of Surgery of Spine and Spinal Cord, Zhengzhou University People's Hospital, \\ Henan Provincial People's Hospital, Zhengzhou, Henan 450003; ${ }^{4}$ Department of Gynecology Oncology, \\ Harbin Medical University Cancer Hospital, Harbin, Heilongjiang 150086; \\ ${ }^{5}$ Department of Surgery of Spine and Spinal Cord, Henan Provincial People's Hospital,
}

Henan International Joint Laboratory of Intelligentized Orthopedics Innovation and Transformation, Henan Key Laboratory for Intelligent Precision Orthopedics, People's Hospital of Zhengzhou University,

People's Hospital of Henan University, Zhengzhou, Henan 450003, P.R. China

Received January 11, 2021; Accepted June 7, 2021

DOI: $10.3892 / \mathrm{mco} .2021 .2333$

\begin{abstract}
In recent years, major discoveries have indicated that Ras homology family member C (RHOC) is involved in the occurrence and pathological progression of a number of malignant tumours; nevertheless, the role served by RHOC in glioma remains unclear. The present study aimed to gain further insight into the biological function and expression of RHOC in human glioma based on the Chinese Glioma Genome Atlas (CGGA). The current study analysed $~ 1,000$ glioma samples from the CGGA. First, RHOC expression was analysed according to the clinical features associated with the prognosis of glioma, such as clinical stage, histological type and age. Second, the Kaplan-Meier method was used, revealing that the survival rate of patients with glioma with high RHOC expression was significantly lower than that of patients with low RHOC expression. Receiver operating characteristic curve analysis indicated that RHOC had moderate diagnostic value for patients with glioma. Gene set enrichment analysis
\end{abstract}

Correspondence to: Professor Yanzheng Gao, Department of Surgery of Spine and Spinal Cord, Henan Provincial People's Hospital, Henan International Joint Laboratory of Intelligentized Orthopedics Innovation and Transformation, Henan Key Laboratory for Intelligent Precision Orthopedics, People's Hospital of Zhengzhou University, People's Hospital of Henan University, 7 Weiwu Road, Zhengzhou, Henan 450003, P.R. China

E-mail: yanzhenggaohn@gs.zzu.edu.cn

*Contributed equally

Key words: Ras homology family member C, glioma, early diagnosis, treatment indirectly indicated that RHOC mainly participated in the pathological mechanism of glioma through p53, extracellular matrix receptor interaction and focal adhesion. Finally, the aforementioned results were further verified using The Cancer Genome Atlas data and reverse transcription-quantitative PCR technology. To the best of our knowledge, the present study was the first comprehensive in-depth analysis of RHOC, revealing the potential value of RHOC as a novel oncogene in glioma. The current study provided a novel potential biomarker for the diagnosis and prognosis of glioma, and re-examined the pathological mechanism of glioma from a new perspective.

\section{Introduction}

Glioma, an intracranial tumour originating from glial cells, is the most common primary intracranial tumour. There are nearly 100,000 newly diagnosed patients worldwide each year (1). Its high disability rate and mortality rate seriously affect people's quality of life and threaten people's lives (2). At present, the diagnosis of gliomas mainly relies on imaging technology. Comprehensive treatment includes extensive surgical resection, adjuvant chemoradiotherapy, immunotherapy and photodynamic therapy $(3,4)$. Although effective interventions have been developed for the occurrence and development of gliomas, the survival rate of glioma patients has not been significantly improved (5). The poor prognosis is mainly due to the stage and the degree of malignancy of the tumour. The 5-year survival time of early cancer patients is relatively long (6). Therefore, it is necessary to look for reliable and sensitive predictors associated with tumour staging and prognosis to evaluate the occurrence and progression of tumours early, further improve the prognosis of patients and prolong the survival time of patients.

Through a large number of literature searches, we have gained a preliminary understanding of the pathological 
mechanism of gliomas and have discovered some biological targets for the diagnosis and treatment of gliomas (7). For example, there are reports that TERT gene mutations can affect the survival time of glioma patients by controlling the interaction between the TERT promoter mutation and IDH mutation in glioma (8). Furthermore, inhibition of the TP53 GOF mutation can inhibit inflammation in glioblastoma multiforme (GBM). Bioinformatics analysis also shows that inducing TP53 GOF mutation upregulation can significantly shorten the survival time of glioma patients (9). EGFR, CCDC26, PHLDB1 and other genes are also involved in the growth and development of glioma (10). These biological targets have been used for the diagnosis and treatment of gliomas. This is mainly because there are many molecular subtypes of gliomas, and the pathological mechanism is more complicated and is not determined by a single factor. Therefore, an in-depth understanding of the complex pathogenesis of gliomas and the discovery of new molecular predictors for gliomas are essential.

Ras homology family member C (RHOC), a vital member of the Rho GTPase family, has various biological functions, such as regulating cytoskeletal reorganization and affecting cell adhesion and migration (11). Some biological functions of RHOC in malignant tumour cells have been explored by many scholars. RHOC plays a potential subtle role in invasive melanoma, gastric cancer, oesophageal squamous cell carcinoma and other malignant tumours by influencing the biological behaviours of malignant tumour cells, such as proliferation and apoptosis (11-13). It has been reported that RHOC knockout can inhibit the invasion and migration of cholangiocarcinoma cells by inhibiting MMP2, MMP3, MMP9 and epithelial-mesenchymal transition (EMT) (14). Similar to this finding, the metastasis and invasion of ovarian cancer were also found to be regulated by MMP9, which was closely related to the significant inhibition of MMP9 expression by RHOC (15). In addition, in oesophageal squamous cell carcinoma (ESCC), the expression of RHOC was positively correlated with the depth of tumour invasion and the degree of lymph node metastasis (16). Although the biological function of RHOC has been explored in many tumour cells, there are few studies on the relationship between RHOC and glioma.

To the best of our knowledge, the expression patterns and molecular functions of RHOC have not been entirely characterized in gliomas. To uncover this information, we used RNA sequencing (RNA-Seq) data from the Chinese Glioma Genome Atlas (CGGA) data set to comprehensively describe the molecular patterns and clinical relevance of RHOC in glioma. At the same time, to make our research results more reliable, we also used the RNA-Seq data of glioma samples from The Cancer Genome Atlas (TCGA) data set as a validation cohort. This comprehensive study is the first to reveal the expression patterns and molecular functions of RHOC in glioma.

\section{Materials and methods}

Data collection. We downloaded 749 RNA-Seq data points of glioma samples from the CGGA database. Additionally, we obtained another 666 glioma tissue RNA-Seq data points from the TCGA database and obtained the clinical information of the corresponding patients. To further verify the credibility of our trial, we collected 5 glioma tissue samples and 5 non-tumour brain tissue samples from daily surgery, stored them in a liquid nitrogen environment, and then transferred them to a $-80^{\circ} \mathrm{C}$ freezer. These 10 samples were used to determine the RHOC expression level by reverse transcription-polymerase chain reaction (RT-PCR). For the sample trial, we obtained written informed consent from the corresponding patients.

Gene set enrichment analysis (GSEA) of RHOC. GSEA is a genetic probe based on the evaluation of data from microarrays. It can be used to determine whether a predetermined gene shows a statistically significant difference between two biological states. We used GSEA to predefine the level of RHOC expression. The genes were clearly divided into the RHOC high expression group and the RHOC low expression group and analysed for statistical significance. The genome was permuted 1000 times per analysis. We considered genes with $\mathrm{P}<0.05$ and false discovery rate (FDR) $<0.25$ to be statistically significant. Finally, we also used the Kyoto Encyclopedia of Genes and Genomes (KEGG) database for enrichment analysis of the data set. After exploring the signal pathway through GSEA and KEGG enrichment analysis, we further explored the coexpression genes related to RHOC gene expression through coexpression analysis, and analyzed the coexpression genes by Pearson's correlation coefficient method. The analysis and mapping were based on R language software (v.3.6.1).

RNA isolation and RT-qPCR analysis. RT-PCR is a technique that combines RNA RT with cDNA PCR. The RNA strand was reverse transcribed into cDNA and then amplified into a DNA template by PCR technology. In our study, total RNA was extracted from gliomas and normal brain tissues, and the total absorbance at $260 \mathrm{~nm}$ was determined by a spectrophotometer. The cDNA was then reverse transcribed from the total RNA using the First Strand cDNA Synthesis Kit (Roche). RT qPCR was performed according to the guidelines of Faststart Universal SYBR Green Master (Rox) (Roche, Germany). The results were quantified using quantum Studio software (Thermo Fisher Scientific, Inc.) according to the manufacturer's instructions. The internal reference was GAPDH, and the primer sequences were 5'-CAAGGTCAT CCATGACAACTTTG-3' (F) and 5'-GTCCACCACCCTGTT GCTGTAG-3' (R). The primer sequences for RHOC were 5'-CCTGAGCCTTGACTTCATCTC-3' (F) and 5'-CCACCT CAATGTCCGCAATA-3' (R). RT-PCR data were analysed by the $\triangle \mathrm{CT}$ method. The paired t-test was used to analyse the two groups of data, and $\mathrm{P}<0.05$ was considered to be statistically significant.

Statistical analysis. R software (v.3.6.1) was used to perform statistical analysis, and the Wilcoxon signed-rank test was used to examine the expression of RHOC in glioma and non-tumour brain tissues. The potential relationship between the expression level of RHOC and the overall survival of glioma patients was analysed using the Cox regression and Kaplan-Meier methods, and the receiver operating characteristic (ROC) curve also confirmed the potential value of RHOC in glioma diagnosis. Univariate and multivariate analyses were used to explore the relationship between the clinical features and overall survival time of glioma patients. Welch's t-test was used to analyze the differences between groups. The Wilcoxon 
Table I. Characteristics of patients with glioma based on the Chinese glioma genome atlas.

\begin{tabular}{|c|c|c|}
\hline Characteristics & Number of cases & Percentages, $\%$ \\
\hline \multicolumn{3}{|l|}{ PRS type } \\
\hline Primary & 502 & 67.02 \\
\hline Recurrent & 222 & 29.64 \\
\hline Secondary & 25 & 3.34 \\
\hline \multicolumn{3}{|l|}{ Histology } \\
\hline A & 55 & 7.34 \\
\hline $\mathrm{AA}$ & 39 & 5.21 \\
\hline $\mathrm{AO}$ & 22 & 2.94 \\
\hline $\mathrm{AOA}$ & 80 & 10.68 \\
\hline GBM & 176 & 23.50 \\
\hline $\mathrm{O}$ & 35 & 4.67 \\
\hline $\mathrm{OA}$ & 95 & 12.68 \\
\hline $\mathrm{rA}$ & 20 & 2.67 \\
\hline rAA & 36 & 4.81 \\
\hline $\mathrm{rAO}$ & 15 & 2.00 \\
\hline rAOA & 48 & 6.41 \\
\hline rGBM & 90 & 12.02 \\
\hline $\mathrm{rO}$ & 4 & 0.53 \\
\hline $\mathrm{rOA}$ & 9 & 1.20 \\
\hline sGBM & 25 & 3.34 \\
\hline \multicolumn{3}{|l|}{ Grade } \\
\hline WHO II & 218 & 29.11 \\
\hline WHO III & 240 & 32.04 \\
\hline WHO IV & 291 & 38.85 \\
\hline \multicolumn{3}{|l|}{ Sex } \\
\hline Male & 307 & 40.99 \\
\hline Female & 442 & 59.01 \\
\hline \multicolumn{3}{|l|}{ Age, years } \\
\hline$\leq 41$ & 343 & 45.79 \\
\hline$>41$ & 406 & 54.21 \\
\hline \multicolumn{3}{|c|}{ Radiotherapy status } \\
\hline Yes & 625 & 83.44 \\
\hline No & 124 & 16.56 \\
\hline \multicolumn{3}{|c|}{ Chemotherapy status } \\
\hline Yes & 520 & 69.43 \\
\hline No & 229 & 30.57 \\
\hline \multicolumn{3}{|c|}{ IDH mutation status } \\
\hline Mutant & 410 & 54.74 \\
\hline Wild-type & 339 & 45.26 \\
\hline \multicolumn{3}{|c|}{$1 \mathrm{p} 19 \mathrm{q}$ codeletion status } \\
\hline Non-codel & 155 & 20.69 \\
\hline Codel & 594 & 79.31 \\
\hline
\end{tabular}

WHO, World Health Organization; IDH, isocitrate dehydrogenase; A, astrocytoma; AA, anaplastic astrocytoma; AO, anaplastic oligodendroglioma; AOA, anaplastic oligoastrocytoma; GBM, glioblastoma multiforme; O, oligodendroglioma; OA, oligoastrocytoma; r, recurrence; sGBM, secondary GBM; PRS type, polygenic risk score type.

rank sum test and Kruskal-Wallis test were used to analyse the association between RHOC expression and clinical features.

\section{Results}

Patient characteristics. We obtained a total of 749 glioma samples from the CGGA database, which contains a variety of complete clinical data, such as polygenic risk score (PRS) type, age, sex, histological type, chemotherapy status, 1p19 codeletion status and IDH mutation status. More detailed clinical feature information is shown in Table I.

Correlation between RHOC expression and clinical characteristics in glioma patients. Our study found that the expression level of RHOC is indeed significantly correlated with age, tumour grade, chemotherapy status, 1p19 codeletion status, IDH mutation status and histological type. The expression of RHOC increases as the tumour grade increases (Fig. 1). The RHOC expression level of patients $<41$ years was significantly lower than that of patients $>41$ years. The expression of RHOC in the 1p19 codeletion and IDH mutant groups was significantly lower than that in the non-1p19 codeletion $(\mathrm{P}<0.001)$ and IDH wild-type $(\mathrm{P}<0.001)$ groups. In addition, we found that the expression levels of RHOC in the GBM and recurrent GBM groups increased significantly.

Survival outcomes and clinical diagnostic value of RHOC in patients with glioma. The Kaplan-Meier survival analysis method explored the potential link between overall survival and RHOC expression levels in glioma patients. As shown in Fig. 2, the analysis of the CGGA database showed that there were obvious abnormalities between the RHOC high expression group and the RHOC low expression group. In terms of survival time, glioma patients with high RHOC expression had significantly worse survival times than those with low RHOC expression. Moreover, the area under the curve (AUC) values for survival in the first year, the third year and the fifth year were $0.725,0.792$ and 0.803 , respectively, which were all $>0.7$. It was further confirmed that RHOC had moderate diagnostic value.

Univariate and multivariate analyses. After univariate analysis by the Cox regression model (shown in Fig. 3A), high RHOC expression, PRS type, histological type, tumour grade, age and receiving chemotherapy were closely associated with the poor prognosis of glioma patients $(\mathrm{P}<0.001)$ [(hazard ratio $(\mathrm{HR})=1.862 ; 95 \%$ confidence interval $(\mathrm{CI}), 1.688-2.053)$, $(\mathrm{HR}=2.123 ; 95 \% \mathrm{CI}, 1.818-2.478),(\mathrm{HR}=4.487 ; 95 \% \mathrm{CI}$, 3.695-5.449), $(\mathrm{HR}=2.883$; 95\% CI, 2.526-3.291), $(\mathrm{HR}=1.624$; 95\% CI, 1.345-1.960), and (HR=1.647; 95\% CI, 1.328-2.044)]. In addition, IDH mutation status and $1 \mathrm{p} 19 \mathrm{q}$ codeletion status were strongly associated with good prognosis $(\mathrm{P}<0.001)$ $[(\mathrm{HR}=0.317 ; 95 \% \mathrm{CI}, 0.262-0.384)$ and $(\mathrm{HR}=0.231 ; 95 \% \mathrm{CI}$, $0.169-0.315)]$.

Multivariate analysis by the Cox regression model (Fig. 3B) showed that high RHOC expression $(\mathrm{P}<0.001)$, PRS type $(\mathrm{P}<0.001)$ and high tumour grade $(\mathrm{P}<0.001)$ were significantly associated with poor prognosis [ $(\mathrm{HR}=1.220$; 95\% CI, 1.088-1.369), (HR=1.992; 95\% CI, 1.696-2.340), and $(\mathrm{HR}=2.628$; 95\% CI, 1.921-3.595)]. However, we also found that glioma patients with IDH mutation status were significantly associated with good prognosis. Moreover, patients with $1 \mathrm{p} 19 \mathrm{q}$ codeletion status were significantly associated 

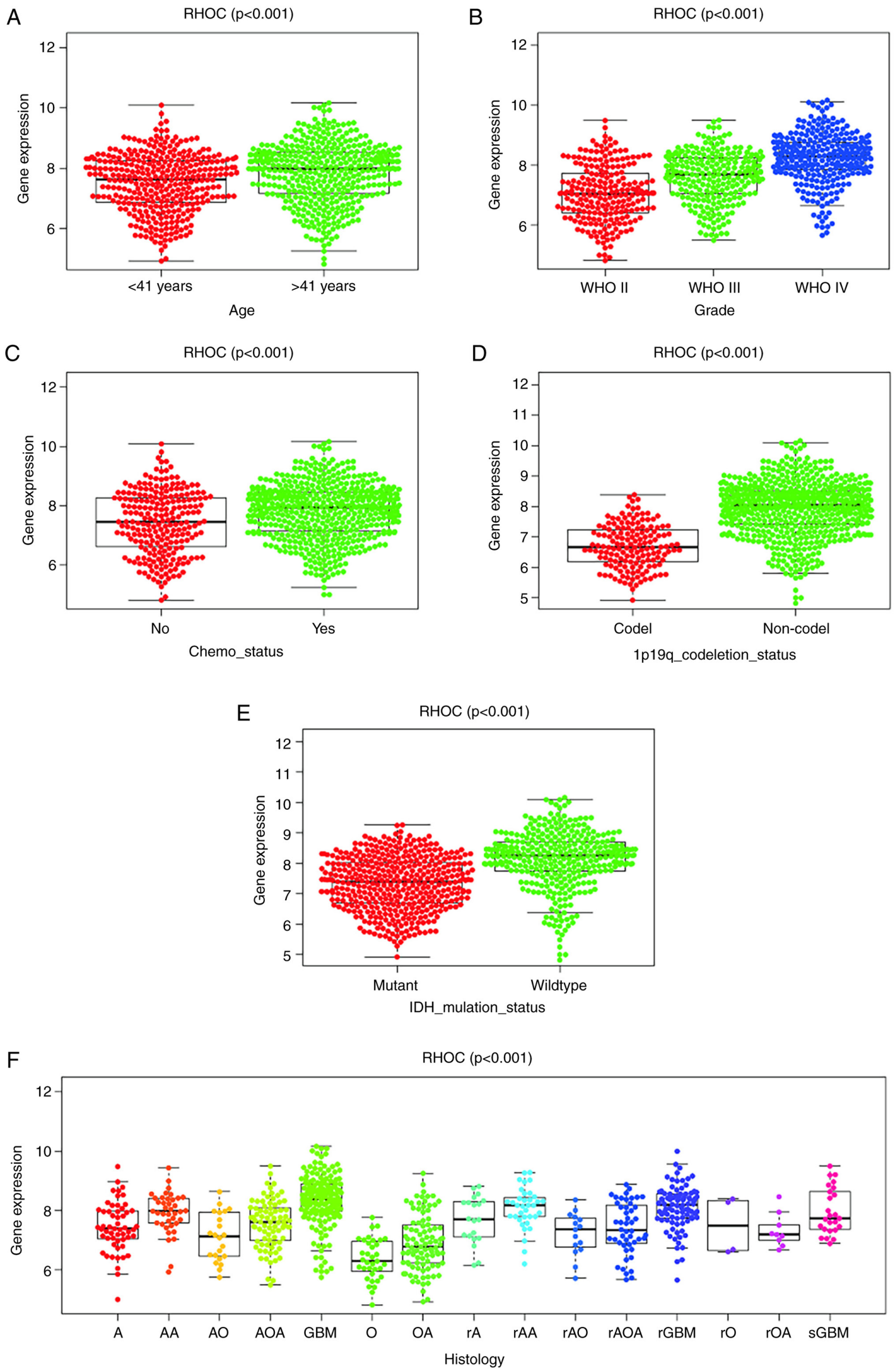

Figure 1. RHOC expression in patients with glioma from the Chinese Glioma Genome Atlas. Association between RHOC expression and clinicopathological characteristics, including (A) age, (B) grade, (C) chemotherapy status, (D) 1p19q codeletion status, (E) IDH mutation status and (F) histology. A, astrocytoma; $\mathrm{AA}$, anaplastic astrocytoma; AO, anaplastic oligodendroglioma; AOA, anaplastic oligoastrocytoma; GBM, glioblastoma multiforme; O, oligodendroglioma; OA, oligoastrocytoma; r, recurrence; sGBM, secondary GBM; RHOC, Ras homology family member C; WHO, World Health Organization; IDH, isocitrate dehydrogenase. 

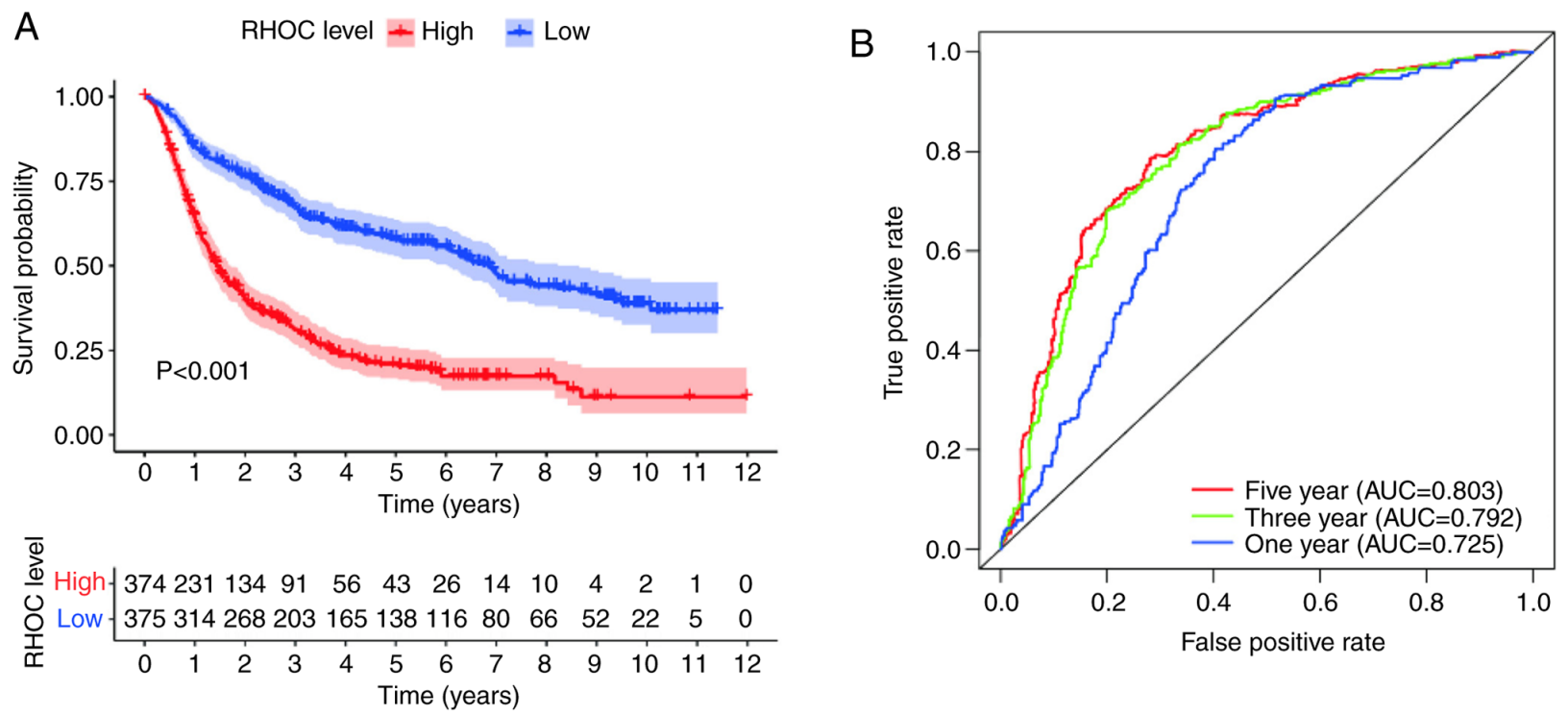

Figure 2. Kaplan-Meier survival curve and ROC curve. (A) High RHOC expression indicated poor overall survival in patients with glioma from the Chinese Glioma Genome Atlas. (B) ROC curve indicated good diagnostic value of RHOC expression in glioma. RHOC, Ras homology family member C; ROC, receiver operating characteristic; AUC, area under the curve.

A

B

$\begin{array}{lc} & \text { P-value } \\ \text { RHOC } & <0.001 \\ \text { PRS_type } & <0.001 \\ \text { Histology } & 0.077 \\ \text { Grade } & <0.001 \\ \text { Sex } & 0.599 \\ \text { Age } & 0.049 \\ \text { Radio } & 0.643 \\ \text { Chemo } & 0.002 \\ \text { IDH_mutation } & <0.001 \\ \text { 1p19q_codeletion } & <0.001\end{array}$

Hazard ratio

$1.862(1.688-2.053)$

$2.123(1.818-2.478)$

4.487 (3.695-5.449)

2.883 (2.526-3.291)

1.044 (0.866-1.258)

1.624 (1.345-1.960)

0.929 (0.720-1.199)

1.647 (1.328-2.044)

0.317 (0.262-0.384)

$0.231(0.169-0.315)$

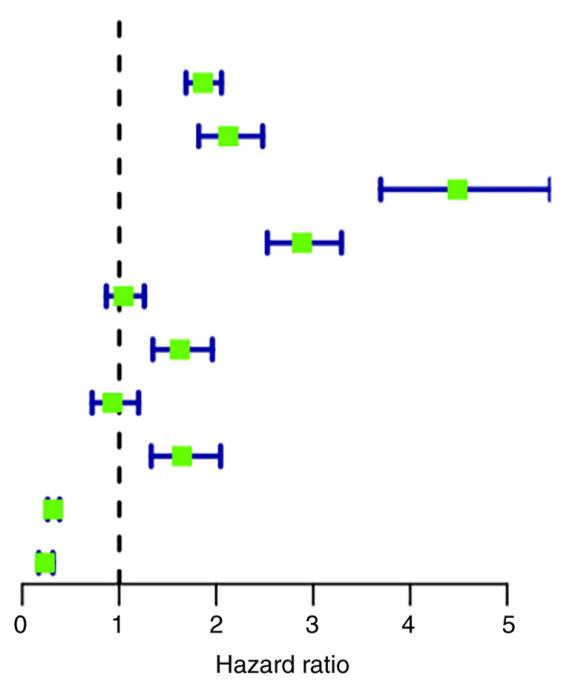

Hazard ratio

$1.220(1.088-1.369)$

1.992 (1.696-2.340)

$0.670(0.430-1.044)$

2.628 (1.921-3.595)

1.053 (0.870-1.274)

1.220 (1.000-1.488)

$0.938(0.716-1.229)$

0.689 (0.543-0.876)

$0.619(0.492-0.780)$

$0.459(0.324-0.649)$

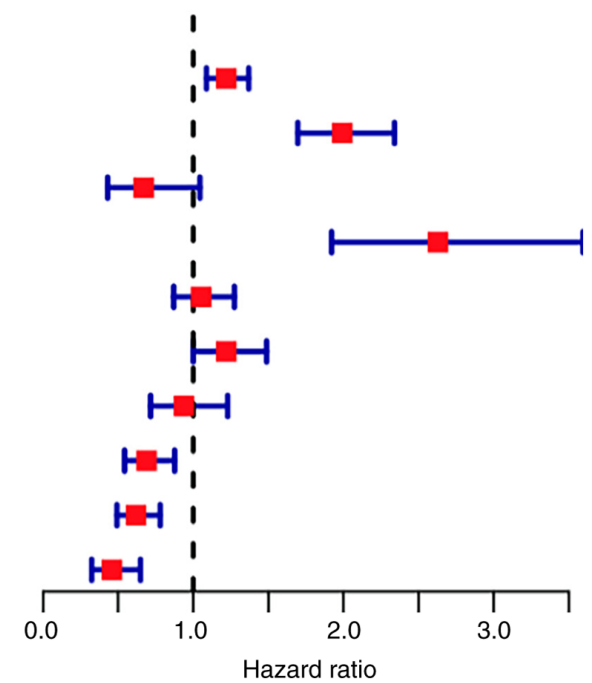

Figure 3. Univariate and multivariate analysis. (A) Univariate regression of prognosis in patients with glioma from the Chinese Glioma Genome Atlas. (B) Multivariate survival model of prognosis in patients with glioma. RHOC, Ras homology family member C; IDH, isocitrate dehydrogenase; PRS type, polygenic risk score type. 
A

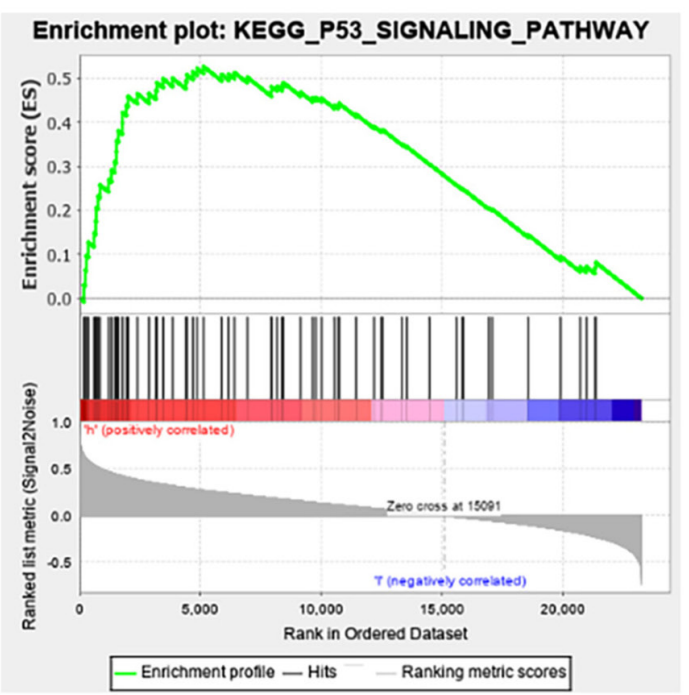

C Enrichment plot: KEGG_ECM_RECEPTOR_INTERACTION

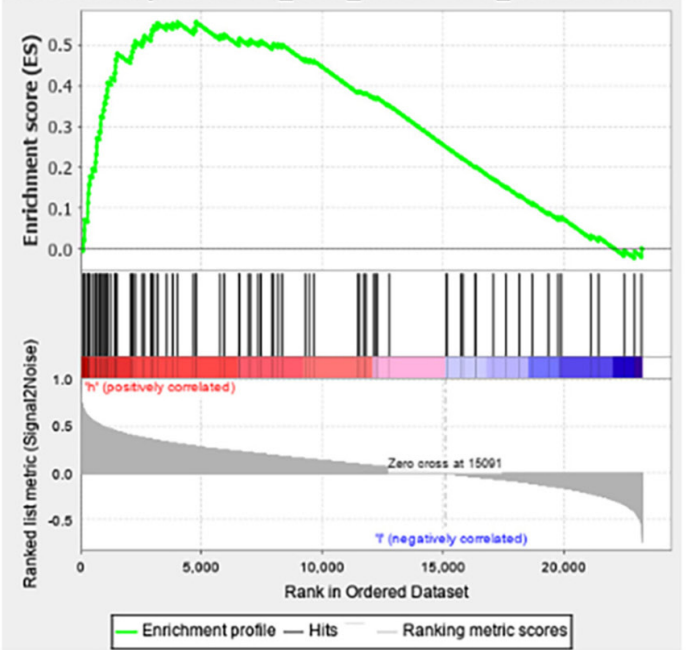

B

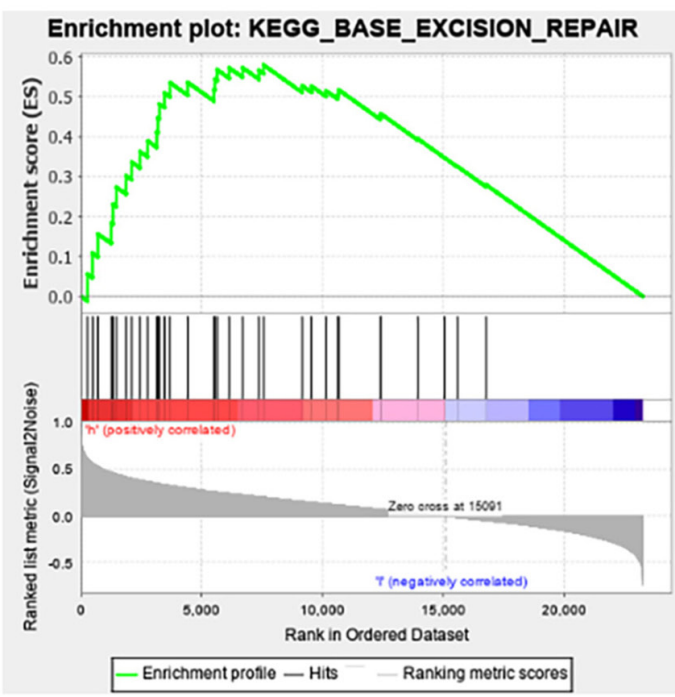

D

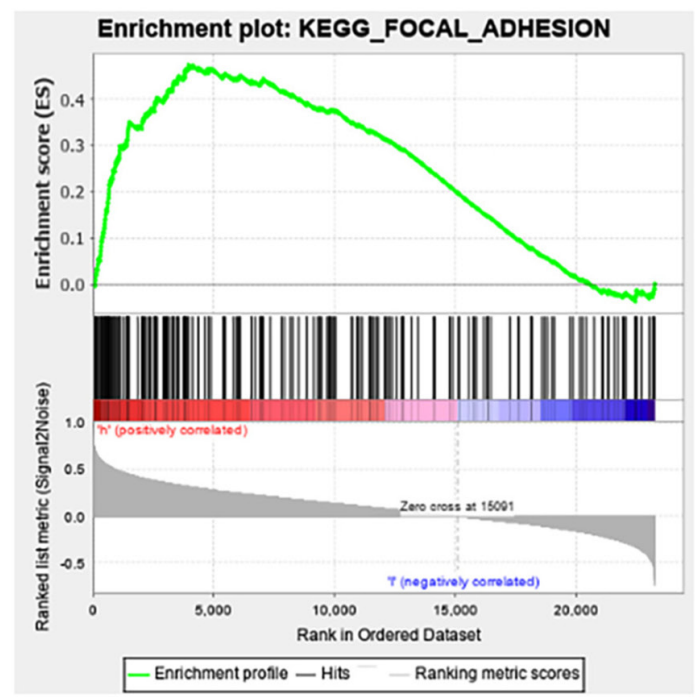

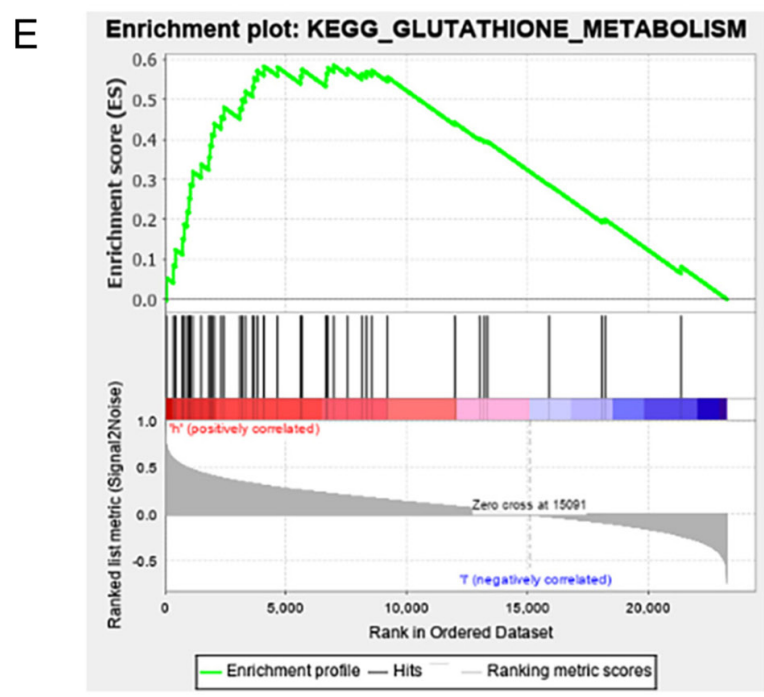

Figure 4. Enrichment plots from Gene Set Enrichment Analysis. (A) P53_SIGNALING_PATHWAY; (B) BASE_EXCISION_REPAIR; (C) ECM_ RECEPTOR_INTERACTIN; (D) FOCAL_ADHESION; (E) GLUTATHIONE_METABOLISM. KEGG, Kyoto Encyclopaedia of Genes and Genomes.

with good prognosis $(\mathrm{P}<0.001)$. Taken together, the univariate and multivariate analyses suggested that the high expression of RHOC might be a poor prognostic factor.
RHOC-related signalling pathways identified by GSEA. As a bioinformatics analysis tool, GSEA analysed the cancer signalling pathways between the RHOC high expression 
Table II. Results from gene set enrichment analysis.

\begin{tabular}{lcc}
\hline Gene set name & NES & NOM P-value \\
\hline $\begin{array}{l}\text { KEGG_P53_SIGNALING_ } \\
\text { PATHWAY }\end{array}$ & 1.6437907 & 0.028 \\
$\begin{array}{l}\text { KEGG_BASE_EXCISION_ } \\
\text { REPAIR }\end{array}$ & 1.6104667 & 0.028 \\
$\begin{array}{l}\text { KEGG_ECM_RECEPTOR_ } \\
\text { INTERACTIN }\end{array}$ & 1.6973425 & 0.039 \\
$\begin{array}{l}\text { KEGG_FOCAL_ADHESION } \\
\text { KEGG_GLUTATHIONE_ } \\
\text { METABOLISM }\end{array}$ & 1.7078017 & 0.033 \\
\hline
\end{tabular}

Gene sets with $|\mathrm{NES}|>1, \mathrm{NOM} \mathrm{P}<0.05$ and false discovery rate q-value $<0.25$ were considered as significantly enriched. NES, normalized enrichment score; NOM, nominal; KEGG, Kyoto Encyclopedia of Genes and Genomes.

Table III. Characteristics of patients with glioma based on the cancer genome atlas.

\begin{tabular}{lcc}
\hline Characteristics & Number of cases & Percentages, \% \\
\hline Sex & & \\
$\quad$ Male & 385 & 57.81 \\
Female & 281 & 42.19 \\
Age, years & & \\
$\quad 51$ & 399 & 59.91 \\
$>51$ & 267 & 40.09 \\
Grade & & \\
WHO II & 243 & 36.49 \\
WHO III & 260 & 39.04 \\
WHO IV & 163 & 24.47 \\
\hline
\end{tabular}

WHO, World Health Organization.

group and the RHOC low expression group. As shown in Fig. 4 and Table II, among all the factors analysed, p53, BASE_EXCISION_REPAIR, ECM receptor, focal adhesion and glutathione metabolism showed significant differential enrichment in the RHOC high expression group. The above results show that RHOC can produce relevant pathological changes in the occurrence and development of glioma through these pathways.

Verifying the RHOC bioinformatic analysis results. To make our findings more credible, the data of a total of 666 glioma patients with complete clinical information and gene expression profile data were retrieved from the TCGA database as shown in Table III. The rnaseq data of 666 gliomas and 5 normal brain tissues were downloaded from TCGA database. Wilcox method was used to detect the expression level of RHOC in gliomas and normal brain tissues. Welch t-test was used to compare the differences between the two groups. The results showed that RHOC was significantly overexpressed in gliomas (Fig. 5A). In addition, we collected 5 glioma tissues and 5 non-tumour tissues from the clinic and detected the expression level of RHOC by RT-PCR technology. The results showed that compared with non-tumour tissues, RHOC was highly expressed in glioma tissues (Fig. 5B). Finally, we derived the overall survival time of glioma patients from the TCGA database and further analysed the data using the Kaplan-Meier method (as shown in Fig. 5C). We found that high RHOC expression indeed leads to poor prognosis in glioma patients. The ROC curve also confirmed the results of previous studies that RHOC has a moderate diagnostic value in glioma (Fig. 5D).

Co-expression network to predict genes that are critical to glioma pathogenesis. We used a heat map to represent the top 20 genes that were most positively and negatively correlated with RHOC expression (Fig. 6C). Co-expression networks consist of gene expression data relative to normal expression levels. In a co-expression network, each node represents one gene in the network, and the gap represents two related genes. String concatenation is a close association between these genes, indicating a possible regulatory relationship. Among them, genes such as NECAP2, AGTRAP, LSM10, PFN1, and AK2 were significantly positively related to RHOC, while genes such as AKT3, RIMS2, ATRNL1, TUB, and RIMS1 were significantly negatively related to RHOC (Fig. 6A). We also tabulated their connection values, as shown in Fig. 6B.

\section{Discussion}

Recently, the role of RHOC has attracted much attention from academia. Numerous studies have shown that RHOC has unique potential value in malignant tumour cells (17). RHOC has been found to be highly expressed in lung cancer, breast cancer, liver cancer and many other tumour tissues and seriously affects the prognosis of patients (18-20). However, few studies have explored the relationship between RHOC and glioma. Therefore, our report will be a pioneering experimental study to clarify the potential diagnostic and prognostic value of RHOC expression in glioma.

Our objective was to investigate the relationship between RHOC and the clinical features of gliomas. We obtained the clinical features of glioma patients from the CGGA database and found that RHOC was highly expressed in glioma. As shown in Fig. 1, the expression level of RHOC was positively correlated with the age of glioma patients and World Health Organization (WHO) grade and had a potential relationship with chemotherapy status, histological type, 1p19q codeletion status and IDH mutation status. The expression level of RHOC was also significantly higher in colorectal cancer tissue cells than in normal and adjacent tissues, and in particular, local intestinal damage in colorectal cancer tissue was also closely associated with the biological function of RHOC (21). Moreover, the invasion and metastasis of hepatocellular carcinoma (HCC) are closely related to the expression and activity of the RHOC protein (22). Some studies have found that RHOC is a key factor in the invasion and metastasis of head and neck squamous cell carcinoma (23). Many studies have shown that the expression level of RHOC is related to the clinical characteristics of malignant tumours. We have reason to believe that the expression level of RHOC also has some 
A

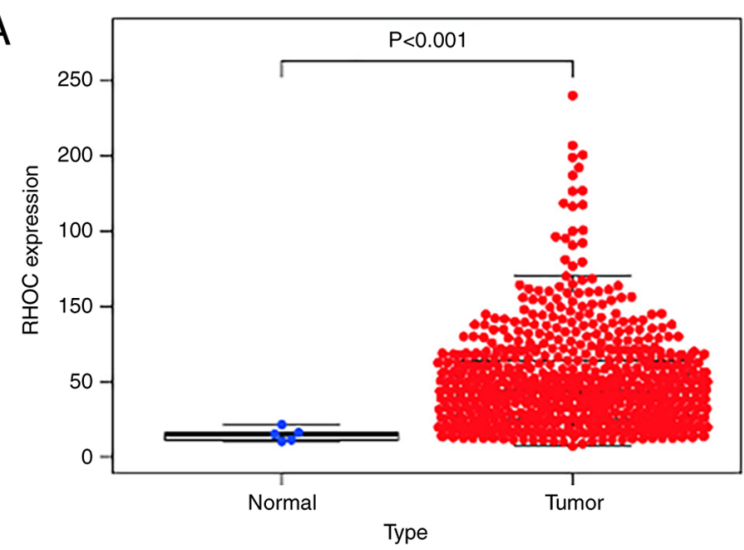

C

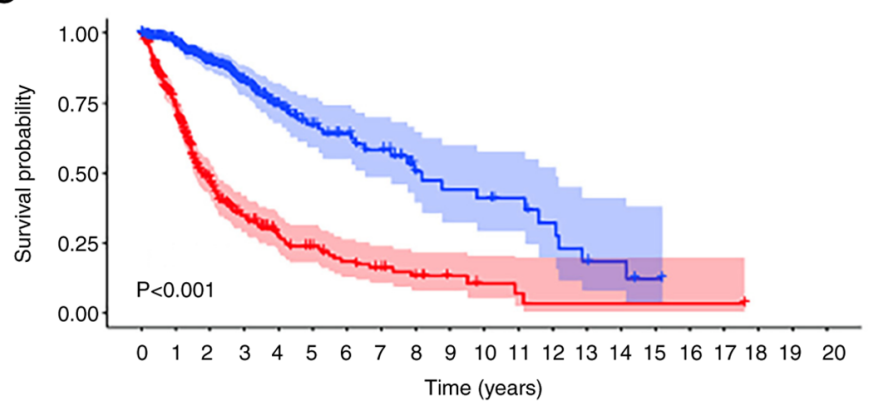

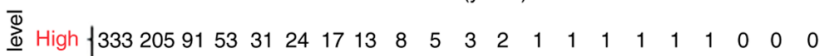
产 Low \begin{tabular}{llllllllllllllllllllllll}
333 & 26417110960 & 42 & 35 & 28 & 16 & 14 & 13 & 10 & 7 & 4 & 3 & 1 & 0 & 0 & 0 & 0 & 0 \\
\hline
\end{tabular} Time (years)
B

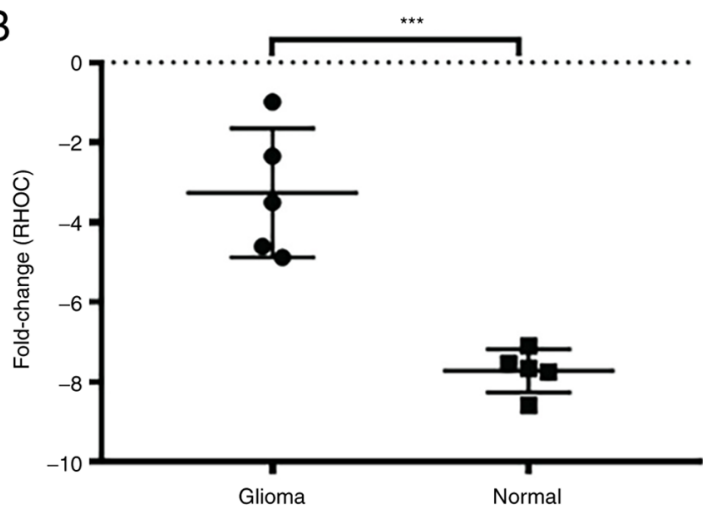

D

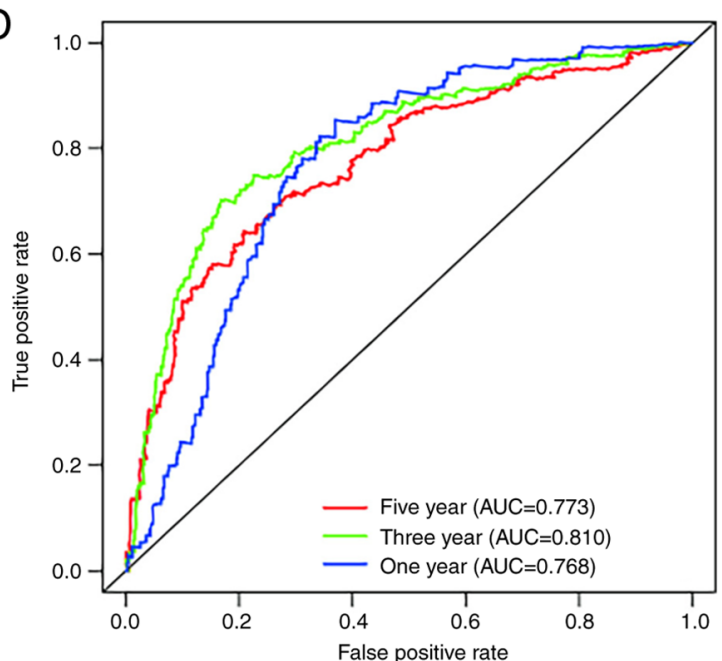

Figure 5. Survival analysis and RT-qPCR verification using TGCA database. (A) RHOC expression was significantly increased in glioma compared with in normal tissues. (B) RT-qPCR verification revealed that RHOC was highly expressed in patients with glioma. (C) Kaplan-Meier curve based on TCGA database demonstrated that high RHOC expression indicated a poor prognosis in patients with glioma. (D) Receiver operating characteristic curve based on TCGA database exhibited good diagnostic value of RHOC expression for glioma. RHOC, Ras homology family member C; AUC, area under the curve; RT-qPCR, reverse transcription-quantitative PCR; TCGA, The Cancer Genome Atlas.

potential correlation with the clinical characteristics of glioma, which was further confirmed by our experimental results.

However, the specific effect of RHOC on glioma cells is still unclear, and we further used the Kaplan-Meier method to analyse the correlation between the RHOC expression level and overall survival of patients. As shown in Fig. 2A, in terms of overall survival, we found that glioma patients with significantly high RHOC expression had better overall survival than those with low RHOC expression. In addition, as shown in Fig. 2B, the ROC curve further confirmed our finding that high RHOC expression leads to poor prognosis in glioma patients, with moderate diagnostic reference value. Studies have shown that overexpression of RHOC may lead to poor prognosis in liver cancer by promoting vasculogenic mimicry (VM) induced by the EMT mechanism (24). Kleer CG found that the expression of RHOC increased with the progression of breast cancer tissue and that high RHOC expression was significantly associated with decreased patient survival (25). To confirm the role of RHOC expression in glioma cells and to further verify whether our findings are inevitable or contingent factors, we performed univariate and multivariate analyses (Fig. 3) and carefully observed that RHOC can indeed serve as an independent risk factor and has moderate diagnostic value.
From the above results, we did not identify the mechanism of RHOC in glioma patients with adverse prognoses. We used GSEA to explore the specific role of RHOC in cell signalling pathways. As shown in Fig. 4, RHOC was significantly enriched in cell signalling pathways (e.g., the p53 signalling pathway, base excision and repair, ECM receptor interaction, focal adhesion, glutathione metabolism). Among them, the ECM receptor interaction pathway, which may be associated with breast cancer, was also reported in previous studies. It has also been found that Twist 2 promotes the proliferation and invasion of renal cell carcinoma (RCC) cells by regulating ECM receptor interactions (26). Most surprisingly, the role of focal adhesion in glioma was also elucidated. HOXA2 was found to be highly expressed in gliomas and could affect the proliferation of gliomas by regulating the focal adhesion pathway (27). CD155/PVR, as a regulatory factor of adhesion signalling, promotes the proliferation of glioma cells by regulating adhesion signalling and local adhesion kinetics (28). Our results also show that RHOC is significantly enriched in the focal adhesion pathway, and it is reasonable to believe that RHOC can affect the occurrence and development of glioma by regulating focal adhesion. 
A
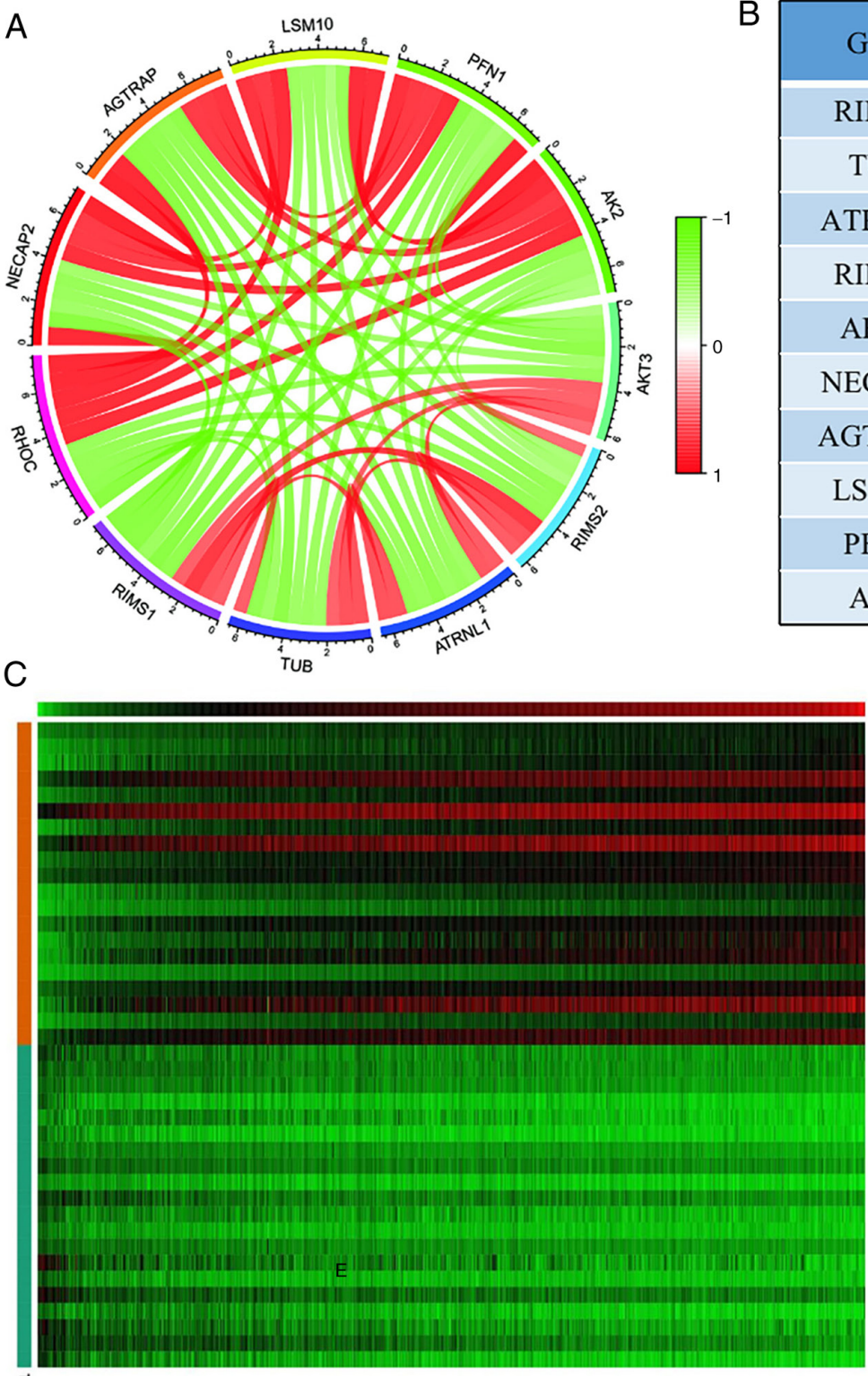

B

\begin{tabular}{|c|c|c|}
\hline Gene & $\begin{array}{c}\text { Correlation } \\
\text { coefficient }\end{array}$ & P-value \\
\hline RIMS1 & -0.735 & $5.67 \mathrm{E}-174$ \\
\hline TUB & -0.683 & $6.29 \mathrm{E}-141$ \\
\hline ATRNL1 & -0.668 & $1.27 \mathrm{E}-132$ \\
\hline RIMS2 & -0.667 & $2.93 \mathrm{E}-132$ \\
\hline AKT3 & -0.643 & $4.56 \mathrm{E}-120$ \\
\hline NECAP2 & 0.867 & $5.89 \mathrm{e}-310$ \\
\hline AGTRAP & 0.862 & $1.46 \mathrm{E}-302$ \\
\hline LSM10 & 0.86 & $7.07 \mathrm{E}-299$ \\
\hline PFN1 & 0.852 & $9.14 \mathrm{E}-288$ \\
\hline AK2 & 0.851 & $1.02 \mathrm{E}-286$ \\
\hline
\end{tabular}

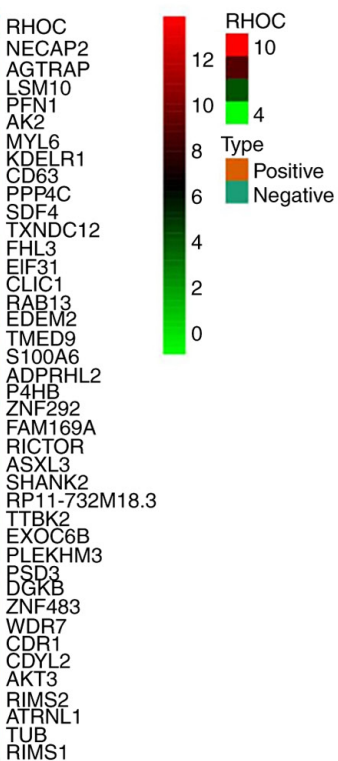

Figure 6. (A) Co-expression of genes with RHOC. (B) Correlation coefficient of co-expression genes. (C) Top 20 genes that were most positively or negatively correlated with RHOC expression represented by a heat map. RHOC, Ras homology family member C.

The occurrence and development of glioma itself is very complex, involving many biological processes not only related to RHOC. Thus, in order to predict the pathogenesis of glioma more accurately, we further conducted co-expression analysis and the results showed that NECAP2, AGTRAP, LSM10, PFN1 and AK2 are conducive to the occurrence and development of glioma. AKT3, RIMS2, ATRNL1, TUB and RIMS1 contribute to prognosis and survival. Previous studies have shown that PFN1 phosphorylation can obviously promote the development of glioma (29), and targeting Akt3 may become an effective method for treating glioma patients (30). Although the remaining eight genes have not currently been found to be significant in glioma by researchers, we strongly believe that they will be studied by scholars in the near future to discover their potential value.

Although we performed an extensive analysis using public databases to gain more insight into the relationship between RHOC and glioma, there are still some limitations.
Our sample came from a public database so it has inherent shortcomings, such as differences in the inclusion criteria, geographical differences, differences in the extent of surgical resection, and differences in the dose of chemoradiotherapy. However, public databases have unique advantages, such as multicentre design, large sample sizes, and ethnic diversity. Second, we used bioinformatics methods to deeply understand the potential link between RHOC and glioma. Because of the complexity of glioma pathogenesis and the wide range of gene functions, this topic still needs to be explored by future scholars to further understand the relationship between RHOC and glioma more comprehensively. Our study provides a basis for future scholars to guide progress in glioma research.

Through a series of analysis methods, this study concluded that the abnormally high expression of RHOC could serve as a novel oncogene independently leading to poor prognosis in glioma. In addition, we identified possible important 
carcinogenic pathways. We firmly believe that RHOC will become a new biomarker for the diagnosis and prognosis of glioma.

\section{Acknowledgements}

Not applicable.

\section{Funding}

The present study was supported by the Central Plains Thousand Talents Plan of Henan Province (grant no. ZYQR201912111).

\section{Availability of data and materials}

All data generated or analyzed during this study are included in this published article.

\section{Authors' contributions}

BZ, JY and XL conceived and designed the experiment. BL provided reagents/analytical tools, performed data analysis and participated in drafting the manuscript. YW and HW have also made contributions to the design of this experiment and part of the data acquisition. JW and MZ have made contributions to data acquisition, data analysis and interpretation. YaZ, YoZ and RL analyzed the data and prepared charts. YG provided the final approval of the released version, and made a contribution to the data analysis and interpretation. BZ, JY and XL confirm the authenticity of all the raw data to ensure its validity. All authors read and approved the final manuscript.

\section{Ethics approval and consent to participate}

All procedures performed in the present study were in accordance with the ethical standards of the Ethical Committee of the Henan Provincial People's Hospital. The experimental scheme was also approved by the Ethics Committee of Henan Provincial People's Hospital [approval no. 2020 lunshenzi (08)]. All patients provided written informed consent.

\section{Patient consent for publication}

Not applicable.

\section{Competing interests}

The authors declare that they have no competing interests.

\section{References}

1. Bray F, Ferlay J, Soerjomataram I, Siegel R, Torre L and Jemal A Global cancer statistics 2018: GLOBOCAN estimates of incidence and mortality worldwide for 36 cancers in 185 countries. CA Cancer J Clin 68: 394-424, 2018.

2. Wang H, Xu T, Huang Q, Jin W and Chen J: Immunotherapy for malignant glioma: Current status and future directions. Trends Pharmacol Sci 41: 123-138, 2020.

3. Kong Z, Yan C, Zhu R, Wang J, Wang Y, Wang Y, Wang R, Feng $\mathrm{F}$ and $\mathrm{Ma} \mathrm{W}$ : Imaging biomarkers guided anti-angiogenic therapy for malignant gliomas. Neuroimage Clin 20: 51-60, 2018.
4. Delgado-López P, Corrales-García E, Martino J, Lastra-Aras E and Dueñas-Polo M: Diffuse low-grade glioma: A review on the new molecular classification, natural history and current management strategies. Clin Transl Oncol 19: 931-944, 2017.

5. Ho VK, Reijneveld JC, Enting RH, Bienfait HP, Robe P, Baumert BG and Visser O; Dutch Society for Neuro-Oncology (LWNO): Changing incidence and improved survival of gliomas. Eur J Cancer 50: 2309-2318, 2014.

6. Okuyama A, Shibata A and Nishimoto H: Critical points for interpreting patients' survival rate using cancer registries: A literature review. J Epidemiol 28: 61-66, 2018.

7. Gao W, Li H,Liu Y,Zhang Y,Zhao H and Liu F: Long non-coding RNA FLVCR1-AS1 promotes glioma cell proliferation and invasion by negatively regulating miR-30b-3p. Mol Med Rep 22: 723-732, 2020

8. Vuong HG, Altibi AMA, Duong UNP, Ngo HTT, Pham TQ, Chan AK, Park CK, Fung KM and Hassell L: TERT promoter mutation and its interaction with IDH mutations in glioma: Combined TERT promoter and IDH mutations stratifies lower-grade glioma into distinct survival subgroups-a metaanalysis of aggregate data. Crit Rev Oncol Hematol 120: 1-9, 2017.

9. Ham SW, Jeon HY, Jin X, Kim EJ, Kim JK, Shin YJ, Lee Y, Kim SH, Lee SY, Seo S, et al: TP53 Gain-of-function mutation promotes inflammation in glioblastoma. Cell Death Differ 26: 409-425, 2019.

10. Ostrom QT, Bauchet L, Davis FG, Deltour I, Fisher JL, Langer CE, Pekmezci M, Schwartzbaum JA, Turner MC, Walsh KM, et al: The epidemiology of glioma in adults: A 'state of the science' review. Neuro Oncol 16: 896-913, 2014.

11. Thomas P, Pranatharthi A, Ross C and Srivastava S: RhoC: A fascinating journey from a cytoskeletal organizer to a cancer stem cell therapeutic target. J Exp Clin Cancer Res 38: 328, 2019.

12. Wu Y, Chen Y, Sang J and Xu W: RhoC protein stimulates migration of gastric cancer cells through interaction with scaffold protein IQGAP1. Mol Med Rep 4: 697-703, 2011.

13. Zhao ZH, Tian Y, Yang JP, Zhou J and Chen KS: RhoC, vascular endothelial growth factor and microvascular density in esophageal squamous cell carcinoma. World J Gastroenterol 21: 905-912, 2015.

14. Yang H, Liang J, Zhou J, Mi J, Ma K, Fan Y, Ning J, Wang C, Wei $\mathrm{X}$ and Li E: Knockdown of RHOC by shRNA suppresses invasion and migration of cholangiocellular carcinoma cells via inhibition of MMP2, MMP3, MMP9 and epithelial-mesenchymal transition. Mol Med Rep 13: 5255-5261, 2016.

15. Zhao Y, Zong ZH and Xu HM: RhoC expression level is correlated with the clinicopathological characteristics of ovarian cancer and the expression levels of ROCK-I, VEGF, and MMP9. Gynecol Oncol 116: 563-571, 2010.

16. Zhang HZ, Liu JG, Wei YP, Wu C, Cao YK and Wang M: Expression of G3BP and RhoC in esophageal squamous carcinoma and their effect on prognosis. World J Gastroenterol 13: 4126-4130, 2007.

17. Lang S, Busch $\mathrm{H}$, Boerries M, Brummer T, Timme S, Lassmann S, Aktories K and Schmidt G: Specific role of RhoC in tumor invasion and metastasis. Oncotarget 8: 87364-87378, 2017.

18. Arisanty D, Harahap W, Khambri D, Rustam R, Aliska G, Achyar A and Menra JP: The comparison of RhoC and PI3K gene expression on the breast cancer tissue and benign tumour tissue. Open Access Maced J Med Sci 7: 1911-1916, 2019.

19. Shen Y, Bu L, Li R, Chen Z, Tian F and Ge Q: Expression and biological interaction network of RHOC for hepatic carcinoma with metastasis in PBMC samples. Onco Targets Ther 12: 9117-9128, 2019.

20. Bellovin DI, Simpson KJ, Danilov T, Maynard E, Rimm DL, Oettgen P and Mercurio AM: Reciprocal regulation of RhoA and RhoC characterizes the EMT and identifies RhoC as a prognostic marker of colon carcinoma. Oncogene 25: 6959-6967, 2006.

21. Wang HB, Liu XP, Liang J, Yang K, Sui AH and Liu YJ: Expression of RhoA and RhoC in colorectal carcinoma and its relations with clinicopathological parameters. Clin Chem Lab Med 47: 811-817, 2009.

22. Xie S, Zhu M, Lv G, Zhang Q and Wang G: The role of RhoC in the proliferation and apoptosis of hepatocellular carcinoma cells. Med Oncol 29: 1802-1809, 2012.

23. Tumur Z, Katebzadeh S, Guerra C, Bhushan L, Alkam T and Henson BS: RhoC mediates epidermal growth factor-stimulated migration and invasion in head and neck squamous cell carcinoma. Neoplasia 17: 141-151, 2015. 
24. Guo JQ, Zheng QH, Chen H, Chen L, Xu JB, Chen MY, Lu D, Wang ZH, Tong HF and Lin S: Ginsenoside Rg3 inhibition of vasculogenic mimicry in pancreatic cancer through downregulation of VE-cadherin/EphA2/MMP9/MMP2 expression. Int J Oncol 45: 1065-1072, 2014

25. Kleer CG, Griffith KA, Sabel MS, Gallagher G, van Golen KL, $\mathrm{Wu}$ ZF and Merajver SD: RhoC-GTPase is a novel tissue biomarker associated with biologically aggressive carcinomas of the breast. Breast Cancer Res Treat 93: 101-110, 2005.

26. Zhang HJ, Tao J, Sheng L, Hu X, Rong RM, Xu M and Zhu TY: Twist 2 promotes kidney cancer cell proliferation and invasion by regulating ITGA6 and CD44 expression in the ECM-receptor interaction pathway. Onco Targets Ther 9: 1801-1812, 2016.

27. Liu Z, Shen F, Wang H, Li A, Wang J, Du L, Liu B, Zhang B, Lian X, Pang B, et al: Abnormally high expression of HOXA2 as an independent factor for poor prognosis in glioma patients. Cell Cycle 19: 1632-1640, 2020.
28. Sloan KE, Stewart JK, Treloar AF, Matthews RT and Jay DG: CD155/PVR enhances glioma cell dispersal by regulating adhesion signaling and focal adhesion dynamics. Cancer Res 65: 10930-10937, 2005.

29. Fan Y, Potdar AA, Gong Y, Eswarappa SM, Donnola S, Lathia JD, Hambardzumyan D, Rich JN and Fox PL: Profilin-1 phosphorylation directs angiocrine expression and glioblastoma progression through HIF-1 $\alpha$ accumulation. Nat Cell Biol 16: 445-456, 2014.

30. Mure H, Matsuzaki K, Kitazato KT, Mizobuchi Y, Kuwayama K, Kageji T and Nagahiro S: Akt2 and Akt3 play a pivotal role in malignant gliomas. Neuro Oncol 12: 221-232, 2010.

(i) $\Theta$ This work is licensed under a Creative Commons Attribution-NonCommercial-NoDerivatives 4.0 International (CC BY-NC-ND 4.0) License. 